\title{
Mapping Quantitative Mechanical Properties at Molecular Scale Using Peak Force Tapping AFM
}

\author{
Chanmin $\mathrm{Su}$ \\ Veeco Instruments, 112 Robin Hill Rd, Santa Barbara, CA 93117
}

Atomic force microscope (AFM) has been used primarily as a topographic imaging tool. On the other hand the force interaction between tip and sample was long sought as a method to provide quantitative mechanical information of materials at nanometer scale, ranging from in-vitro biologic material to polymer blends ${ }^{1}$. The method, namely the force spectra, is limited to an individual point measurement of tip-surface interaction. Recent development based on Tapping Mode ${ }^{\mathrm{TM}}$ allowed two dimensional mapping of mechanical property by reconstructing force spectra using tapping harmonics ${ }^{2}$. While tapping harmonics enabled high resolution mechanical mapping, the interaction control is based on tapping amplitude and shares all challenges of Tapping Mode ${ }^{\mathrm{TM}}$, such as fluid operation and interaction force control at sub-nanonewton range.

The new technology, peak force tapping (PFT), operates at sub-resonance of AFM probes and controls the instantaneous interaction force directly. In this technology the feedback setpoint is the peak interaction force each time the tip taps the sample. The operating frequency is high enough so that the peak force feedback has the similar imaging bandwidth as Tapping Mode ${ }^{\mathrm{TM}}$. More importantly the measurement calculates modulus, deformation (hardness), adhesion and energy dissipation through force spectra at each pixel and presents a set of quantitative nanomechanical data concurrent with the topographic imaging process.

To further explore the quantitative nanomechanical mapping a series of benchmark material was developed and used to exam the accuracy of the peak force tapping. The data are shown in figure 1. All the samples are homogeneous materials. The moduli measured by nanomechanical mapping with peak force tapping are compared with known modulus using other methods, ranging from $0.6 \mathrm{MPa}$ to $70 \mathrm{GPa}$.

By using probes of different spring constant, PFT controls interaction force accurately from Piconewtons to Micronewtons. At the Piconewtons level the sharpness of the tip is well preserved, enabling molecular resolution on soft materials consistently. Figure 2 shows simultaneous topographic and nanomechanical mapping of a DNA network. Further study also demonstrated molecular resolution of OmpG membrane, resolving open structure of the molecule at moderate $\mathrm{pH}$ level. The same peak force control also shows consistent tip sharpness by maintaining tip radius below $5 \mathrm{~nm}$ even on a rough Ti coating, known as Tipcheck sample, for hundreds of images. In addition to these examples, the paper also presents extensive experiment data of inhomogeneous materials, including styrene-butadiene co-polymer and poly(n-butylacrylate) molecular brushes where mechanical properties are mapped at individual component of the molecules.

In conclusion, peak force tapping enables quantitative mechanical mapping at nanometer scale for a broad range of materials. Through direct control of the peak force, tip apex is preserved for high resolution imaging on both soft and hard materials. 
${ }^{1}$ Engel A; Gaub H E; Müller D J., Biophysical Journal 76(3) (1999) 1659

${ }^{2}$ Ozgur Sahin, Sergei Magonov, Chanmin Su, Calvin F. Quate \& Olav Solgaard Nature Nanotechnology 2 (2007) 507

\begin{tabular}{|c|c|c|}
\hline Sample & Expec & Meas. \\
\hline DP150 & 0.63 & 0.76 \\
\hline DP130 & 0.73 & 0.87 \\
\hline DP110 & 0.86 & 1.2 \\
\hline DP60 & 1.58 & 2.2 \\
\hline DP25 & 3.78 & 4.1 \\
\hline DPB & 11.8 & 14.5 \\
\hline LDPE9O & 60 & 58 \\
\hline LDPE93 & 400 & 370 \\
\hline Silk & 2400 & 2200 \\
\hline PS Film & 2700 & 2500 \\
\hline HOPG & 10500 & 12800 \\
\hline Au Foil & 77200 & 50200 \\
\hline $\mathrm{SiO} 2$ & 71700 & 59400 \\
\hline
\end{tabular}

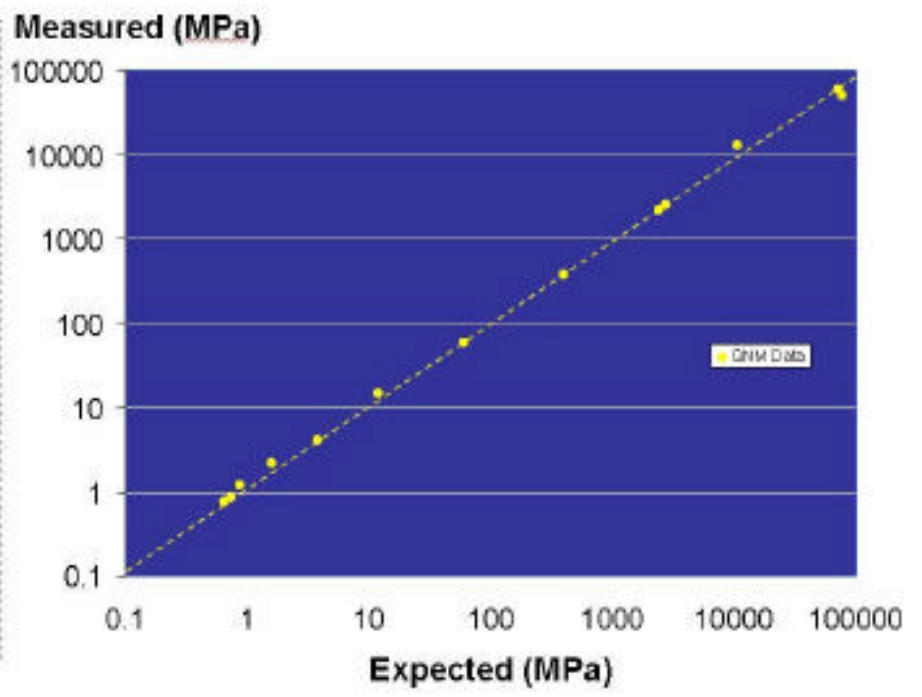

Fig. 1. Quantitative measurement of material modulus using peak force tapping. Table on the left gives the measured in comparison to the expected value of modulus. DP8-DP150 represents PDMS with different degree of polymerization. LDPE stands for low density polyethylene. SiLK is a trade mark of Dow Chemical. PS represents polystyrene. (Data courtesy of Natalia Erina)

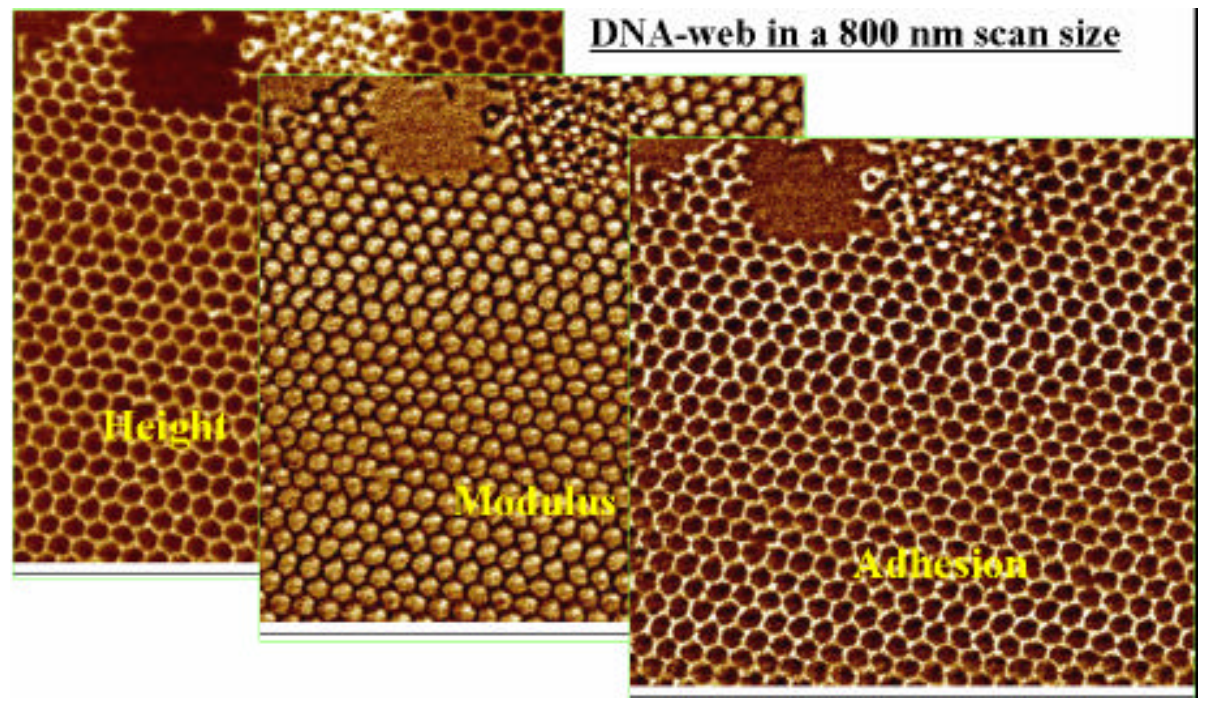

Figure 2. Simultaneous topographic imaging and mechanical mapping of DNA network. (Data courtesy of Natalia Erina and Alex Koyfman) 